\title{
DISCONJUGACY OF COMPLEX DIFFERENTIAL SYSTEMS AND EQUATIONS
}

\author{
BY \\ DAVID LONDON AND BINYAMIN SCHWARZ
}

1. Introduction. In this paper we consider linear differential systems of the form

$$
w^{\prime}(z)=A(z) w(z) .
$$

Here $w(z)$ is the column vector $\left[w_{1}(z), \ldots, w_{n}(z)\right]$ and $A(z)$ is the $n \times n$ matrix $\left(a_{i k}(z)\right)_{1}^{n}$, where the $n^{2}$ analytic functions $a_{i k}(z)$ are regular in a bounded simply connected domain $D$. Together with the vector differential equation (1), we consider also the corresponding matrix differential equation

$$
W^{\prime}(z)=A(z) W(z),
$$

where $W(z)=\left(w_{i k}(z)\right)_{1}^{n}$. In [12], (1) was called disconjugate in $D$ if, for every choice of $n$ (not necessarily distinct) points $z_{1}, \ldots, z_{n}$ in $D$, the only solution of (1) which satisfies $w_{i}\left(z_{i}\right)=0, i=1, \ldots, n$, is the trivial one $w(z) \equiv 0$. Disconjugacy of (1) in $D$ is equivalent to the fact that for any fundamental solution $W(z)=\left(w_{i k}(z)\right)_{1}^{n}$ of (2) (i.e., for any solution $W(z)$ for which the determinant $|W(z)|=\left|w_{i k}(z)\right|_{1}^{n} \neq 0$ for all $z$ of $D$ ), the determinant $\left|w_{i k}\left(z_{i}\right)\right|_{1}^{n} \neq 0$ for every choice of $n$ (not necessarily distinct) points $z_{1}, \ldots, z_{n}$ of $D$ [12, Theorem 3]. (If this holds for one fundamental solution of (2), then it holds for all of them.) This property may thus serve as the definition of disconjugacy of the systems (2), and (1), in $D$.

We define now a more restrictive property than this above defined (ordinary) disconjugacy in $D$.

DefinItion 1. The differential systems (1) and (2) are called $z_{0}$-absolute disconjugate in $D$ if there exists a point $z_{0} \in D$ such that the solution $W(z)=\left(w_{i k}(z)\right)_{i}^{n}$ of (2), determined by

$$
W\left(z_{0}\right)=I \quad\left(\text { i.e., } w_{i k}\left(z_{0}\right)=\delta_{i k}, i, k=1, \ldots, n\right),
$$

satisfies

$$
\lambda\left(\left(\left|w_{i k}\left(z_{i k}\right)-\delta_{i k}\right|\right)_{1}^{n}\right)<1,
$$

for every choice of $n^{2}$ (not necessarily distinct) points $z_{i k}$ in $D . \lambda(A)=\lambda\left(\left(a_{i k}\right)_{1}^{n}\right)$ denotes the (Perron-Frobenius) maximal characteristic value of the nonnegative matrix $A=\left(a_{i k}\right)_{1}^{n}\left(A\right.$ is nonnegative if $a_{i k} \geqq 0$ for all $\left.i, k=1, \ldots, n\right)$.

Received by the editors September 25, 1967. 
In $\$ 2$ we give sufficient conditions ensuring the $z_{0}$-absolute disconjugacy of (2). These results (Theorems 1-3) rely on simple properties of characteristic values of matrices (Lemmas 1 and 3), and on the Peano-Baker method of solution of differential systems. From Lemma 2 follows that $z_{0}$-absolute disconjugacy implies (ordinary) disconjugacy, so each of these theorems yields a sufficient condition for (ordinary) disconjugacy. As shown at the end of $\$ 3$, ordinary disconjugacy does in general not imply $z_{0}$-absolute disconjugacy.

While for general systems (1) and (2) the main consequence of $z_{0}$-absolute disconjugacy is (ordinary) disconjugacy, the situation is different in the special case where

$$
A(z)=\left(\begin{array}{cccccc}
0 & 1 & 0 & & \cdots & 0 \\
0 & 0 & 1 & 0 & \cdots & 0 \\
\vdots & \vdots & & & \vdots \\
0 & 0 & \cdots & 0 & 1 \\
-a_{0}(z) & -a_{1}(z) & \cdots & & & -a_{n-1}(z)
\end{array}\right)
$$

The column vector $w(z)=\left[w_{1}(z), \ldots, w_{n}(z)\right]$ of (1) becomes now $\left[y(z), y^{\prime}(z), \ldots\right.$, $\left.y^{(n-1)}(z)\right]$ and (1) is equivalent to the differential equation

$$
y^{(n)}(z)+a_{n-1}(z) y^{(n-1)}(z)+\cdots+a_{0}(z) y(z)=0 .
$$

$W(z)$ of (2) becomes the Wronskian matrix of $n$ solutions of (4). If $A(z)$ is given by (3), then ordinary disconjugacy of (1) and (2) in $D$ is equivalent to the property that, for every choice of $n$ (not necessarily distinct) points $z_{1}, \ldots, z_{n}$ in $D$, the only solution of (4) satisfying $y\left(z_{1}\right)=y^{\prime}\left(z_{2}\right)=\cdots=y^{(n-1)}\left(z_{n}\right)=0$ is the trivial one $y(z) \equiv 0$. Using a term due to Nehari [9], see also [6], we call this property disfocality of the equation (4) in $D$. As $z_{0}$-absolute disconjugacy implies ordinary one, it follows that if $A(z)$ is given by (3), then $z_{0}$-absolute disconjugacy of the systems (1) and (2) in $D$ implies disfocality of the equation (4) in $D$.

In $\S 3$, using Hermite's formula for divided differences, we prove that if the domain $D$ is convex, then $z_{0}$-absolute disconjugacy in $D$ of the systems (1) and (2), with $A(z)$ given by (3), implies not only disfocality of (4) but also strong disconjugacy of (4) in $D$ (Theorem 4). This term will be defined later (Definition 2). We note here only that strong disconjugacy of (4) in $D$ includes both disfocality and disconjugacy of (4) in $D$. (We use the term "disconjugacy of (4) in $D$ " in the by now classical sense: No (nontrivial) solution $y(z)$ of (4) has more than $n-1$ zeros in $D$, where the zeros are counted with their multiplicities.)

In $\S 4$ we apply Theorem 4 to the results of $\S 2$ and obtain thus sufficient conditions for the equation (4) to be strong disconjugate in a convex domain (Theorems $\left.1^{\prime}-3^{\prime}\right)$.

In $\S 5$ we bring a nonoscillation theorem for equation (4) in the unit disk (Theorem 5). 
This paper is, to a certain degree, a continuation of [12], but we have tried to make it selfcontained. It is again strongly influenced by many of the methods and results of Nehari [7]-[9].

2. Sufficient conditions for $z_{0}$-absolute disconjugacy of systems. We first bring some lemmas on characteristic values needed for the results of this section. We use standard notation for $n \times n$ matrices with (constant) complex elements: $A=\left(a_{i k}\right)_{1}^{n}, B=\left(b_{i k}\right)_{1}^{n}, I=\left(\delta_{i k}\right)_{1}^{n} . A \ll B$ means $\left|a_{i k}\right| \leqq b_{i k} ; i, k=1, \ldots, n$. If $A=\left(a_{i k}\right)_{1}^{n}$, then $A^{+}=\left(\left|a_{i k}\right|\right)_{1}^{n}$, while $|A|=\left|a_{i k}\right|_{1}^{n}$ is the determinant of $A . \lambda_{i}(A), i=1, \ldots, n$, are the $n$ characteristic values of $A=\left(a_{i k}\right)_{1}^{n}$ and, as stated before, $\lambda(A)=\lambda\left(\left(a_{i k}\right)_{1}^{n}\right)$ is the maximal characteristic value of the nonnegative matrix $A=\left(a_{i k}\right)_{1}^{n}$ (i.e., $\left|\lambda_{i}(A)\right| \leqq \lambda(A), i=1, \ldots, n$, and $\lambda(A)$ is one of the $\left.\lambda_{i}(A)\right)$.

We quote now the following well-known result of the Perron-Frobenius theory.

LEMMA 1. If $A \ll B$, then $\left|\lambda_{i}(A)\right| \leqq \lambda(B), i=1, \ldots, n$.

See [3, p. 57], and for a very simple proof see [11, p. 82]. We shall also need two simple consequences of this lemma.

LEMMA 2. If $\lambda\left((A-I)^{+}\right)<1$, then $|A| \neq 0$.

Proof. Lemma 1 implies $\left|\lambda_{i}(A-I)\right|<1, i=1, \ldots, n$. As $\lambda_{i}(A-I)=\lambda_{i}(A)-1$, it follows that $\left|\lambda_{i}(A)-1\right|<1$ and thus $\lambda_{i}(A) \neq 0, i=1, \ldots, n$. Hence, $|A| \neq 0$.

It follows from Lemma 2 that $z_{0}$-absolute disconjugacy of the system (2) in $D$ implies its (ordinary) disconjugacy there. Indeed, by (b) of Definition 1,

$$
\lambda\left(\left(\left|w_{i k}\left(z_{i k}\right)-\delta_{i k}\right|\right)_{1}^{n}\right)<1
$$

for any $n^{2}$ points $z_{11}, \ldots, z_{n n}$ in $D$. Lemma 2 thus gives $\left|w_{i k}\left(z_{i k}\right)\right|_{1}^{n} \neq 0$, where $W(z)=\left(w_{i k}(z)\right)_{1}^{n}$ is the fundamental solution of (2) satisfying $W\left(z_{0}\right)=I$. This implies $\left|w_{i k}\left(z_{i}\right)\right|_{1}^{n} \neq 0$ for any $n$ points $z_{1}, \ldots, z_{n}$ in $D$, which is the definition of (ordinary) disconjugacy of (2) in $D$.

LEMMA 3. If $(A-I) \ll(B-I)$ and $\lambda(B)<2$, then $\lambda\left((A-I)^{+}\right)<1$.

Proof. It follows from the first assumption that $B-I$ is nonnegative. Assume now, by contradiction, that $\lambda(B-I) \geqq 1$. Then there would exist a characteristic value $\lambda_{i}(B)$ such that $\lambda_{i}(B)-1=\lambda(B-I) \geqq 1$, i.e., $\lambda_{i}(B) \geqq 2$. But this contradicts our second assumption. Hence, $\lambda(B-I)<1$, and the conclusion follows by Lemma 1 .

After these preparations we now state

THeOReM 1. Let the bounded domain $D$ be starlike with respect to its point $z_{0}$, and assume that the analytic functions $a_{i k}(z), i, k=1, \ldots, n$, are regular and bounded in $D$. Let $r=\sup \left|z-z_{0}\right|, z \in D$. For each $x, 0 \leqq x<r$, denote by $C(x)$ the intersection of the circle $\left|z-z_{0}\right|=x$ and $D$. Set

$$
m_{i k}(x)=\sup _{z \in C(x)}\left|a_{i k}(z)\right|, \quad i, k=1, \ldots, n, \quad 0 \leqq x<r .
$$


Let the nonnegative functions $p_{i k}(x), i, k=1, \ldots, n$, be continuous in $0 \leqq x \leqq r$, and satisfy

$$
p_{i k}(x) \geqq m_{i k}(x), \quad i, k=1, \ldots, n, \quad 0 \leqq x<r .
$$

Let $U(x)=\left(u_{i k}(x)\right)_{i}^{n}, 0 \leqq x \leqq r$, be the fundamental solution of the real differential system

$$
U^{\prime}(x)=P(x) U(x), \quad 0 \leqq x \leqq r, \quad P(x)=\left(p_{i k}(x)\right)_{i}^{n}
$$

satisfying the initial condition

$$
U(0)=I
$$

If

$$
\lambda(U(r))<2
$$

then the complex differential system

$$
W^{\prime}(z)=A(z) W(z),
$$

$\left(A(z)=\left(a_{i k}(z)\right)_{1}^{n}\right)$, is $z_{0}$-absolute disconjugate in $D$. The number 2 on the right-hand side of (9) cannot be replaced by any larger number.

Proof. Let $W(z)$ be the fundamental solution of (2) in $D$ satisfying the initial condition

$$
W\left(z_{0}\right)=I \text {. }
$$

By the Peano-Baker (matricant) method of solution [5, p. 408]; [3, p. 136], we have for any $z \in D$

$$
W(z)=I+\int_{z_{0}}^{z} A(\zeta) d \zeta+\int_{z_{0}}^{z} A(\zeta) \int_{z_{0}}^{\zeta} A\left(\zeta_{1}\right) d \zeta_{1} d \zeta+\cdots,
$$

where the integrals are taken along the segments from $z_{0}$ to $z$, from $z_{0}$ to $\zeta$, etc. By our assumptions, (11) converges uniformly and absolutely in $D$. For each element $w_{i k}(z), i, k=1, \ldots, n$, of $W(z)$ and for each $z \in D$ we thus obtain

$$
w_{i k}(z)-\delta_{i k}=\int_{z_{0}}^{z} a_{i k}(\zeta) d \zeta+\sum_{j=1}^{n} \int_{z_{0}}^{z} a_{i j}(\zeta) \int_{z_{0}}^{\zeta} a_{j k}\left(\zeta_{1}\right) d \zeta_{1} d \zeta+\cdots
$$

Similarly it follows that for $0 \leqq x \leqq r$ the solution $U(x)$, satisfying (8), of the majorant system (7) is given by

$$
U(x)=I+\int_{0}^{x} P(\xi) d \xi+\int_{0}^{x} P(\xi) \int_{0}^{\xi} P\left(\xi_{1}\right) d \xi_{1} d \xi+\cdots .
$$

For each element $u_{i k}(x), i, k=1, \ldots, n$, of $U(x)$ and for each $x, 0 \leqq x \leqq r$, we thus obtain

$$
u_{i k}(x)-\delta_{i k}=\int_{0}^{x} p_{i k}(\xi) d \xi+\sum_{j=1}^{n} \int_{0}^{x} p_{i j}(\xi) \int_{0}^{\xi} p_{j k}\left(\xi_{1}\right) d \xi_{1} d \xi+\cdots
$$


We choose now $n^{2}$, not necessarily distinct, points $z_{i k}$ in $D$ and set $x_{i k}=\left|z_{i k}-z_{0}\right|$, $i, k=1, \ldots, n$. Using (5), (6), (11') and (12'), we obtain

$$
\left|w_{i k}\left(z_{i k}\right)-\delta_{i k}\right| \leqq u_{i k}\left(x_{i k}\right)-\delta_{i k}, \quad i, k=1, \ldots, n .
$$

But each element $u_{i k}(x)$ of $U(x)$ is, by $\left(12^{\prime}\right)$ and the nonnegativity of $P(x)$, a nonnegative nondecreasing function of $x, 0 \leqq x \leqq r$. (13) thus implies

$$
\left|w_{i k}\left(z_{i k}\right)-\delta_{i k}\right| \leqq u_{i k}(r)-\delta_{i k}, \quad z_{i k} \in D, \quad i, k=1, \ldots, n .
$$

Having chosen arbitrarily the $n^{2}$ points $z_{i k}$, let us denote $w_{i k}\left(z_{i k}\right)=\tilde{w}_{i k}, i, k=1, \ldots, n$; i.e., we set

$$
\tilde{W}=\left(w_{i k}\left(z_{i k}\right)\right)_{1}^{n}
$$

With this notation, (14) becomes

$$
(\tilde{W}-I) \ll(U(r)-I) .
$$

This and assumption (9) imply, by Lemma 3, that

$$
\lambda\left((\tilde{W}-I)^{+}\right)<1 .
$$

(10), (15), and (17) show that (2) is $z_{0}$-absolute disconjugate in $D$.

To prove the last (sharpness) statement of the theorem, we consider the real majorant system (7) on $[0, r]$. Let $U(x), 0 \leqq x \leqq r$, as before, be the solution of (7) satisfying (8). Using the obvious definition of $x_{0}$-absolute disconjugacy of a real system in a segment containing $x_{0}$, it follows that if $\lambda(U(r))>2$, then (7) is not 0absolute disconjugate in $[0, r)$. Indeed, by continuity, we can find $\varepsilon, 0<\varepsilon<r$, such that $\lambda(U(r-\varepsilon))>2$. Hence, $\lambda(U(r-\varepsilon)-I)>1$. Let now the $n^{2}$ points $x_{i k}$ coincide with $r-\varepsilon$. Then $\tilde{U}=\left(u_{i k}\left(x_{i k}\right)\right)_{1}^{n}=U(r-\varepsilon)$ and thus $\lambda(\tilde{U}-I)>1$.

Let now $A(z)$ be a constant nonnegative matrix, $A(z)=A=\left(a_{i k}\right)_{1}^{n}$, and assume that the domain $D$ (contained in $\left|z-z_{0}\right|<r$ ) contains the horizontal segment $\left[z_{0}, z_{0}+r\right)$. Choosing now $P(x)=A$, clearly $W\left(z_{0}+x\right)=U(x), 0 \leqq x<r$. (Here $W(z)$ is the solution of (2) satisfying (10), and $U(x)$ is the solution of (7) satisfying (8).) Choosing all $z_{i k}=z_{0}+r-\varepsilon, \lambda(U(r))>2$ implies $\lambda\left((\tilde{W}-I)^{+}\right)>1$, and (2) is thus not $z_{0}$-absolute disconjugate in $D$. This completes the proof of Theorem 1 .

We add two remarks.

(i) There exist systems (2) and domains $D$ such that (2) is $z_{0}$-absolute disconjugate in $D$ for a certain $z_{0} \in D$, but such that in every neighborhood of $z_{0}$ there are points $z^{*}$ for which (2) is not $z^{*}$-absolute disconjugate in $D$. To show this, let the constant matrix $A(z)=A$ of the above example be positive and assume, as before, that $D$ contains the segment $\left[z_{0}, z_{0}+r\right)$. Setting again $P(x)=A$, it follows that now $\lambda(U(r))=2$ implies $z_{0}$-absolute disconjugacy in $D$. (We have now strict inequality in (14) for each $i, k$.) Continuing the system (7) to the right of $x=r$, it follows that for every $\delta>0, \lambda(U(r+\delta))>2$. If we now choose $z^{*}=z_{0}-\delta$ then, by the above considerations, (2) is not $z^{*}$-absolute disconjugate in $D$. 
(ii) In the general case, assumption (9) is equivalent to the statement that the real majorant system ( 7 ) is 0 -absolute disconjugate in $[0, r]$. Theorem 2 could thus be formulated as a comparison theorem.

To apply Theorem 1, we have to solve the system (7) or we have, at least, to find an estimate for $U(r)$ which ensures (9). In the remaining two theorems of this section we shall define nonnegative matrices, depending in a more direct way on $A(z)$, such that an inequality for their maximal characteristic values implies $z_{0^{-}}$ absolute disconjugacy of (2) in $D$.

THEOREM 2. Let the bounded domain $D$ be starlike with respect to its point $z_{0}$, and let $r=\sup \left|z-z_{0}\right|, z \in D$. Assume that the analytic functions, $a_{i k}(z), i, k=1$, $\ldots, n$, are regular and bounded in $D$ and denote

$$
\alpha_{i k}=\sup _{z \in D}\left|a_{i k}(z)\right|, \quad i, k=1, \ldots, n .
$$

Let $A=\left(\alpha_{i k}\right)_{1}^{n}$ and assume that

$$
r \lambda(A)<\log 2 .
$$

Then the differential system (2) is $z_{0}$-absolute disconjugate in $D$. The number $\log 2$ on the right-hand side of (19) cannot be replaced by any larger number.

Proof. Theorem 2 follows easily from Theorem 1 . Indeed, choosing for $P(x)$ of Theorem 1 the constant matrix $A=\left(\alpha_{i k}\right)_{1}^{n}$, the solution of the corresponding system (7), satisfying (8), is given by

$$
U(x)=e^{x A}=I+x A+\left(x^{2} / 2 !\right) A^{2}+\cdots .
$$

As

$$
\lambda(U(r))=\lambda\left(e^{r A}\right)=e^{r \lambda(A)},
$$

(19) is equivalent to (9), and so (2) is $z_{0}$-absolute disconjugate in $D$.

Considering again the cases where $A(z)=A=\left(\alpha_{i k}\right)_{1}^{n}$ is a constant nonnegative matrix and $\left[z_{0}, z_{0}+r\right)$ is contained in $D$, the sharpness statement follows as in the proof of Theorem 1 .

We add some remarks.

(i) Let us keep the notation and assumptions, except (19), of Theorem 2, but assume, in addition, that the domain $D$ is convex with diameter $d$ and circumcenter $z_{0}$. Then

$$
d \lambda(A)<\sqrt{ } 3 \log 2=1.2005 \cdots
$$

implies the $z_{0}$-absolute disconjugacy of (2) in $D$.

This follows from (19) and the well-known relation $r \leqq d / \sqrt{ } 3$ between the circumradius $r$ and the diameter $d$ of a plane convex set [2, p. 111]. For $z_{0}$-absolute disconjugacy (but probably not for ordinary disconjugacy) $\sqrt{ } 3 \log 2$ on the righthand side of (21) is the best possible number. This follows again by taking for $A(z)$ 
any nonnegative constant matrix and choosing for $D$ an equilateral triangle with center at $z_{0}$ and with one of its vertices at $z_{0}+r$. (21) improves a former result stating that $d \lambda(A)<1$ implies ordinary disconjugacy of (2) in $D$ [12, Theorem 1].

(ii) The starlikeness of the bounded domain $D$ with respect to $z_{0}$ was used only to obtain an upper bound $r$ for the lengths of all paths used in the expansion (11). Theorem 2 remains correct if we only assume on $D$ that there exists a point $z_{0} \in D$ and a positive number $r$, such that every point $z \in D$ can be joined to $z_{0}$ by a path in $D$ of length smaller than $r$.

(iii) We know that $z_{0}$-absolute disconjugacy of the system (2) in $D$ implies its ordinary disconjugacy there. We give now three examples in which we are able to find (or estimate) the radius of 0 -absolute disconjugacy $r_{a}$ (i.e., (2) is 0 -absolute disconjugate in the disk $|z|<r_{a}$, but not in any larger disk) and the radius of ordinary disconjugacy $r_{d}$. In all these examples $A(z)$ will be a constant matrix.

(a) $A(z)=A=\left(\alpha_{i k}\right)_{1}^{n}$ is a constant nonnegative triangular matrix with at least one positive element in its diagonal. ( $\alpha_{i k} \geqq 0$ for all $i, k ; \alpha_{i k}=0$ for $k<i$, and at least one $\alpha_{i i}$ is positive.) Clearly $\lambda(A)=\max \alpha_{i i}>0$ and by Theorem 2 it follows that $r_{a} \geqq$ $(\log 2) / \lambda(A)$. The proof of the sharpness statement shows that $r_{a}=(\log 2) / \lambda(A)$. The fundamental solution $W(z)=\left(w_{i k}(z)\right)_{1}^{n}$ satisfying $W(0)=I$ is also triangular with $w_{i i}(z)=\exp \left(\alpha_{i i} z\right)$. Hence, for any $n$ points $z_{i},\left|w_{i k}\left(z_{i}\right)\right|_{1}^{n} \neq 0$. The system is thus disconjugate in the whole plane, that is $r_{d}=\infty$.

Moreover, while (2) is not 0 -absolute disconjugate for any disk of radius $r>r_{d}$, we have that for any $n^{2}$ points $z_{i k},\left|w_{i k}\left(z_{i k}\right)\right|_{1}^{n} \neq 0$. This shows that in general, $\left|w_{i k}\left(z_{i k}\right)\right|_{1}^{n} \neq 0$, for all choices of $n^{2}$ points in $D$, does not imply $z_{0}$-absolute disconjugacy of (2) in $D$. (Here $W(z)=\left(w_{i k}(z)\right)_{1}^{n}$ is the solution of (2) satisfying (10).)

$$
A(z)=\left(\begin{array}{ll}
1 / 2 & 1 / 2 \\
1 / 2 & 1 / 2
\end{array}\right) ; \quad \lambda(A)=1 \quad \text { and } \quad r_{a}=\log 2
$$

The solution $W(z)$ of (2), satisfying $W(0)=I$, is

$$
W(z)=1 / 2\left(\begin{array}{ll}
e^{z}+1 & e^{z}-1 \\
e^{z}-1 & e^{z}+1
\end{array}\right)
$$

$\left|w_{i k}\left(z_{i}\right)\right|_{1}^{2}=(1 / 2)\left(\exp \left(z_{1}\right)+\exp \left(z_{2}\right)\right) \cdot\left|w_{i k}\left(z_{i}\right)\right|_{1}^{2}=0$ implies $\exp \left(z_{1}-z_{2}\right)=-1$, which gives $r_{d}=\pi / 2$.

$$
A(z)=\left(\begin{array}{rr}
0 & 1 \\
-1 & 0
\end{array}\right) ; \text { hence, } \quad A=\left(\begin{array}{ll}
0 & 1 \\
1 & 0
\end{array}\right) \quad \text { and } \quad \lambda(A)=1 .
$$

By Theorem $2, r_{a} \geqq \log 2=0.693 \cdots$. The solution $W(z)$ of (2), satisfying $W(0)=I$, is

$$
W(z)=\left(\begin{array}{rr}
\cos z & \sin z \\
-\sin z & \cos z
\end{array}\right)
$$


Let $x$ be real and choose $z_{11}=z_{22}=x, z_{12}=-z_{21}=i x$. Then

$$
\tilde{W}(x)=\left(w_{i k}\left(z_{i k}\right)\right)_{1}^{2}=\left(\begin{array}{cc}
\cos x & i \sinh x \\
-i \sinh x & \cos x
\end{array}\right)
$$

and $|\tilde{W}(x)|=\cos ^{2} x-\sinh ^{2} x$. For $x_{0}=0.703 \cdots,\left|\tilde{W}\left(x_{0}\right)\right|=0$ and $\lambda\left(\left(\tilde{W}\left(x_{0}\right)-I\right)^{+}\right)$ $=1$. Hence, $r_{a} \leqq x_{0}$. As $\left|w_{i k}\left(z_{i}\right)\right|_{1}^{2}=\cos \left(z_{1}-z_{2}\right)$, it follows that $r_{d}=\pi / 4=0.785 \cdots$. We conclude this section with the following theorem.

THEOREM 3. Let the bounded domain $D$ be starlike with respect to its point $z_{0}$, and let the analytic functions $a_{i k}(z), i, k=1, \ldots, n$, be regular in $D$. For any $z \in D$, let $\int_{z_{0}}^{z}\left|a_{i k}(\zeta) d \zeta\right|$ be taken along the segment from $z_{0}$ to $z$, and assume that these integrals are bounded uniformly in $z(z \in D)$. Denote

$$
c_{i k}=\sup _{z \in D} \int_{z_{0}}^{z}\left|a_{i k}(\zeta) d \zeta\right|, \quad i, k=1, \ldots, n,
$$

and let $C=\left(c_{i k}\right)_{1}^{n}$. If

$$
\lambda(C)<1 / 2,
$$

then the differential system (2) is $z_{0}$-absolute disconjugate in $D$.

Proof. Using (22) we obtain the following bounds for the absolute values of the terms on the right-hand side of $\left(11^{\prime}\right), z \in D$.

$$
\begin{aligned}
&\left|\int_{z_{0}}^{z} a_{i k}(\zeta) d \zeta\right| \leqq c_{i k}, \quad i, k=1, \ldots, n \\
&\left|\sum_{j=1}^{n} \int_{z_{0}}^{z} a_{i j}(\zeta) \int_{z_{0}}^{\zeta} a_{j k}\left(\zeta_{1}\right) d \zeta_{1} d \zeta\right| \leqq \sum_{j=1}^{n} \int_{z_{0}}^{z}\left|a_{i j}(\zeta)\right| \int_{z_{0}}^{\zeta}\left|a_{j k}\left(\zeta_{1}\right) d \zeta_{1}\right||d \zeta| \\
& \leqq \sum_{j=1}^{n} \int_{z_{0}}^{z}\left|a_{i j}(\zeta) d \zeta\right| \int_{z_{0}}^{z}\left|a_{j k}\left(\zeta_{1}\right) d \zeta_{1}\right| \\
& \leqq \sum_{j=1}^{n} c_{i j} c_{j k}, \quad i, k=1, \ldots, n .
\end{aligned}
$$

Similarly it follows for the $(l+1)$ th term on the right-hand side of $\left(11^{\prime}\right)$,

$$
\left|\sum_{j_{1}, \ldots, j_{l}=1}^{n} \int_{z_{0}}^{z} a_{i j_{1}}(\zeta) \int_{z_{0}}^{\zeta} a_{j_{1} j_{2}}\left(\zeta_{1}\right) \cdots \int_{z_{0}}^{\zeta_{l-1}} a_{j_{l} k}\left(\zeta_{l}\right) d \zeta_{l} \cdots d \zeta_{1} d \zeta\right|
$$

$$
\leqq \sum_{j_{1}, \ldots, j_{l}=1}^{n} c_{i j_{1}} c_{j_{1} j_{2}} \cdots c_{j_{l} k}, \quad i, k=1, \ldots, n .
$$

Let now $\hat{C}=\left(\hat{c}_{i k}\right)_{1}^{n}$ be defined by

$$
\hat{C}=\sum_{l=1}^{\infty} C^{l}=C(I-C)^{-1}
$$


where convergence of the series follows from (23). $\left(11^{\prime}\right),(25)$ and the inequalities (24) give

$$
\left|w_{i k}(z)-\delta_{i k}\right| \leqq \hat{c}_{i k}, \quad z \in D, \quad i, k=1, \ldots, n .
$$

Choosing now $n^{2}$ points $z_{i k}$ in $D$ and using the notation (15), we obtain

$$
(\tilde{W}-I) \ll \hat{C} \text {. }
$$

By $(25), \lambda(\hat{C})=\lambda(C) /(1-\lambda(C))$; hence it follows from (23) that

$$
\lambda(\hat{C})<1 \text {. }
$$

(27) and (28) give, by Lemma 1, (17), and Theorem 3 is thus proved.

From the proof of the sharpness of Theorem 2, it follows that the number $1 / 2$ on the right-hand side of (23) cannot be replaced by any number larger than $\log 2$.

Using the notation (and assumptions) of Theorem 1, we obtain that, in the special case when $c_{i k}=\int_{0}^{r} m_{i k}(x) d x, i, k=1, \ldots, n$, Theorem 1 (with $p_{i k}(x)=m_{i k}(x)$, $i, k=1, \ldots, n)$ is stronger than Theorem 3. Similarly, if $r \alpha_{i k} \leqq(2 \log 2) c_{i k}, i, k=1$, $\ldots, n$, then Theorem 2 is stronger than Theorem 3. However, in the general case, Theorem 3 seems not to be comparable with either one of the former theorems.

3. Strong disconjugacy of differential equations. We begin with the following definition.

DEFINITION 2. The differential equation (4) is called strong disconjugate in $D$ if, for every choice of $n$ (not necessarily distinct) points $z_{1}, \ldots, z_{n}$ in $D$ and every set of natural numbers $k_{1}, \ldots, k_{l}$ such that $k_{1}+\cdots+k_{l}=n$, the only solution of (4) which satisfies

$$
\begin{aligned}
y\left(z_{1}\right) & =\cdots=y\left(z_{k_{1}}\right)=y^{\left(k_{1}\right)}\left(z_{k_{1}+1}\right)=\cdots=y^{\left(k_{1}\right)}\left(z_{k_{1}+k_{2}}\right) \\
& =\cdots=y^{\left(k_{1}+\cdots+k_{l-1}\right)}\left(z_{k_{1}+\cdots+k_{l-1}+1}\right)=\cdots=y^{\left(k_{1}+\cdots+k_{l-1}\right)}\left(z_{n}\right)=0,
\end{aligned}
$$

is the trivial one $y(z) \equiv 0$. (If a value $z^{*}$ appears $m$ times as the argument of the same derivative of order $k_{1}+\cdots+k_{p}$, then this value $z^{*}$ is a zero of $y^{\left(k_{1}+\cdots+k_{p}\right)}(z)$ of at least multiplicity $m$.)

We add some remarks.

(i) For $n=2$ there are 2 possibilities for (29):

$$
y\left(z_{1}\right)=y\left(z_{2}\right)=0, \quad y\left(z_{1}\right)=y^{\prime}\left(z_{2}\right)=0 .
$$

For $n=3$, we have 4 possibilities:

$$
\begin{array}{ll}
y\left(z_{1}\right)=y\left(z_{2}\right)=y\left(z_{3}\right)=0, & y\left(z_{1}\right)=y\left(z_{2}\right)=y^{\prime \prime}\left(z_{3}\right)=0, \\
y\left(z_{1}\right)=y^{\prime}\left(z_{2}\right)=y^{\prime}\left(z_{3}\right)=0, & y\left(z_{1}\right)=y^{\prime}\left(z_{2}\right)=y^{\prime \prime}\left(z_{3}\right)=0 .
\end{array}
$$

In general, for an $n$th order differential equation, (29) includes $2^{n-1}$ cases.

(ii) Strong disconjugacy includes both disfocality (for $l=n, k_{1}=k_{2}=\cdots=k_{n}=1$ ) and disconjugacy (for $l=1, k_{1}=n$ ).

(iii) The equation $y^{(n)}(z)=0$ is strong disconjugate in the whole plane. Indeed, the general solution of $y^{(n)}(z)=0$ is the class $P_{n-1}$ of all the polynomials of degree 
at most $n-1$. Assume that $y(z) \in P_{n-1}$ and satisfies (29) with arbitrary $z_{1}, \ldots, z_{n}$. To prove that $y(z) \equiv 0$, we use induction on $n$. As $k_{1}=n$ implies $y(z) \equiv 0$, we may assume that $k_{1}<n$. Using the $n-k_{1}$ last equations of (29) with respect to $y^{\left(k_{1}\right)}(z)$ $\in P_{n-k_{1}-1}$ and the induction hypothesis, it follows that $y^{\left(k_{1}\right)}(z) \equiv 0$. Hence, $y(z) \in$ $P_{k_{1}-1}$. Using now the $k_{1}$ first equations of (29), it follows that $y(z) \equiv 0$.

It can be shown that if $m_{1}, \ldots, m_{n} ; 0 \leqq m_{1} \leqq m_{2} \leqq \cdots \leqq m_{n}$, are integers such that

$$
y^{\left(m_{i}\right)}\left(z_{i}\right)=0, \quad i=1, \ldots, n,
$$

is different from any one of the $2^{n-1}$ cases included in (29), then there exist $z_{1}, \ldots$, $z_{n}$ and a $y(z) \not \equiv 0$ belonging to $P_{n-1}$ for which $\left(29^{\prime}\right)$ holds.

$y^{(n)}(z)=0$ is thus strong disconjugate in the whole plane, but this would not have remained true if we had added more cases of type $\left(29^{\prime}\right)$. This shows that the above definition of strong disconjugacy is a quite natural one.

(iv) Let (4) be strong disconjugate in $D$, let $z_{1}, \ldots, z_{n}$ and $k_{1}, \ldots, k_{l}$ be as in Definition 2 and let $y_{1}, \ldots, y_{n}$ be arbitrary numbers. Then there exists a unique solution of (4) which satisfies

$$
\begin{aligned}
y\left(z_{1}\right) & =y_{1}, \ldots, y\left(z_{k_{1}}\right)=y_{k_{1}}, \\
y^{\left(k_{1}\right)}\left(z_{k_{1}+1}\right) & =y_{k_{1}+1}, \ldots, y^{\left(k_{1}\right)}\left(z_{k_{1}+k_{2}}\right) \\
& =y_{k_{1}+k_{2}}, \ldots, y^{\left(k_{1}+\cdots+k_{l-1}\right)}\left(z_{k_{1}+\cdots+k_{l-1}+1}\right) \\
& =y_{k_{1}+\cdots+k_{l-1}+1}, \ldots, y^{\left(k_{1}+\cdots+k_{l-1}\right)}\left(z_{n}\right)=y_{n} .
\end{aligned}
$$

Applying the last result to the equation $y^{(n)}(z)=0$, we obtain the existence and uniqueness of an interpolation polynomial for various types of polynomial interpolation determined by (30). (The case $l=1, k_{1}=n$ corresponds to Lagrange interpolation; $l=n, k_{1}=\cdots=k_{n}=1$ corresponds to interpolation in the sense of Abel-Gontscharoff [1, p. 28].)

We bring now the basic theorem which asserts that $z_{0}$-absolute disconjugacy of a system, corresponding to an equation, implies strong disconjugacy of the equation.

TheOREM 4. Let $D$ be a bounded convex domain, and assume that the analytic functions $a_{l}(z), l=0, \ldots, n-1$, are regular in $D$. Let $z_{0} \in D$. If the differential systems (1) and (2), with $A(z)$ given by (3), are $z_{0}$-absolute disconjugate in $D$, then the corresponding differential equation (4) is strong disconjugate in D.

Proof. We shall first prove that if (1) and (2) are $z_{0}$-absolute disconjugate then (4) is disconjugate. This is the part of our assertion corresponding to the case $l=1, k_{1}=n$ of (29).

Consider the systems (1) and (2) with $A(z)$ given by (3). The vector $w(z)=$ $\left[w_{1}(z), \ldots, w_{n}(z)\right]$ becomes now $\left[y(z), y^{\prime}(z), \ldots, y^{(n-1)}(z)\right]$. Let $y_{k}(z), k=1, \ldots, n$, be the solution of (4) satisfying

$$
y_{k}^{(i-1)}\left(z_{0}\right)=\delta_{i k}, \quad i, k=1, \ldots, n .
$$


Let $W(z)$ be the $n \times n$ matrix defined by

$$
W(z)=\left(w_{i k}(z)\right)_{1}^{n}=\left(y_{k}^{(i-1)}\right)_{1}^{n} .
$$

$W(z)$ is the solution of (2) satisfying $W\left(z_{0}\right)=I$. As (2) is $z_{0}$-absolute disconjugate, we have that for every choice of $n^{2}$ points $z_{i k}$ of $D$

$$
\lambda\left(\left(\left|y_{k}^{(i-1)}\left(z_{i k}\right)-\delta_{i k}\right|\right)_{1}^{n}\right)<1 .
$$

We have to show that no nontrivial solution $y(z)$ of (4) can have $n$ zeros $z_{1}, \ldots, z_{n}$ in $D$. By a standard application of Rouché's theorem, it suffices to prove this for distinct zeros $z_{i} \neq z_{j}, i, j=1, \ldots, n$. As every solution $y(z)$ is a linear combination of our $n$ solutions $y_{k}(z)$, it is sufficient to prove that the determinant of the matrix

$$
\Omega=\left(\begin{array}{ccc}
y_{1}\left(z_{1}\right) & \cdots & y_{n}\left(z_{1}\right) \\
y_{1}\left(z_{2}\right) & \cdots & y_{n}\left(z_{2}\right) \\
\vdots & & \vdots \\
y_{1}\left(z_{n}\right) & \cdots & y_{n}\left(z_{n}\right)
\end{array}\right)
$$

is different from zero for any choice of $n$ distinct points $z_{i}$ of $D$.

We now subtract the first row of $\Omega$ from all the other rows and multiply row $i, i=2, \ldots, n$, by $1 /\left(z_{i}-z_{1}\right)$. In the new matrix we subtract row 2 from row $3, \ldots, n$ and multiply by $1 /\left(z_{i}-z_{2}\right)$. Continuing in this fashion, we obtain the matrix

$$
\Omega_{1}=\left(\begin{array}{lll}
y_{1}\left(z_{1}\right) & \cdots & y_{n}\left(z_{1}\right) \\
y_{1}\left[z_{1}, z_{2}\right] & \cdots & y_{n}\left[z_{1}, z_{2}\right] \\
\vdots & & \vdots \\
y_{1}\left[z_{1}, \ldots, z_{n}\right] & \cdots & y_{n}\left[z_{1}, \ldots, z_{n}\right]
\end{array}\right),
$$

where $y\left[z_{1}, \ldots, z_{k}\right]$ denotes the $(k-1)$ th divided difference of $y(z)$ defined by

$$
\begin{aligned}
y\left[z_{1}, z_{2}\right] & =\left(y\left(z_{1}\right)-y\left(z_{2}\right)\right) /\left(z_{1}-z_{2}\right), \\
y\left[z_{1}, \ldots, z_{k}\right] & =\frac{y\left[z_{1}, \ldots, z_{k-1}\right]-y\left[z_{2}, \ldots, z_{k}\right]}{z_{1}-z_{k}}, \quad k=3, \ldots, n .
\end{aligned}
$$

Multiply now the $k$ th $k=1, \ldots, n$, row of $\Omega_{1}$ by $(k-1)$ ! and denote the corresponding matrix by $\tilde{Y}=\left(\tilde{y}_{i k}\right)_{1}^{n}$. That is

$$
\tilde{Y}=\left(\begin{array}{ccc}
y_{1}\left(z_{1}\right) & \cdots & y_{n}\left(z_{1}\right) \\
y_{1}\left[z_{1}, z_{2}\right] & \ldots & y_{n}\left[z_{1}, z_{2}\right] \\
\vdots & & \vdots \\
(k-1) ! y_{1}\left[z_{1}, \ldots, z_{k}\right] & \cdots & (k-1) ! y_{n}\left[z_{1}, \ldots, z_{k}\right] \\
\vdots & & \vdots \\
(n-1) ! y_{1}\left[z_{1}, \ldots, z_{n}\right] & \cdots & (n-1) ! y_{n}\left[z_{1}, \ldots, z_{n}\right]
\end{array}\right) .
$$

As the determinants $|\Omega|$ and $|\tilde{Y}|$ vanish together, it is sufficient to prove that $|\tilde{Y}| \neq 0$. 
We now prove that there exist $z_{i k} \in D$ such that

$$
\left|\tilde{y}_{i k}-\delta_{i k}\right| \leqq\left|y_{k}^{(i-1)}\left(z_{i k}\right)-\delta_{i k}\right|, \quad i, k=1, \ldots, n .
$$

For $i=1 ; k=1, \ldots, n,(32)$ holds trivially for $z_{1 k}=z_{1}$. Now take any element $\tilde{y}_{i k}, i=2, \ldots, n ; k=1, \ldots, n, i \neq k$. Then

$$
\tilde{y}_{i k}=(i-1) ! y_{k}\left[z_{1}, \ldots, z_{i}\right]=\lambda y_{k}^{(i-1)}\left(z_{i k}\right), \quad|\lambda| \leqq 1 .
$$

Here we used a consequence of Hermite's formula for divided differences [10, p. 9]; $\left[8\right.$, p. 328]. $z_{i k}$ belongs to the convex hull of $z_{1}, \ldots, z_{i}$ and so, as $D$ is convex, to $D$. From (33) follows that

$$
\left|\tilde{y}_{i k}\right| \leqq\left|y_{k}^{(i-1)}\left(z_{i k}\right)\right|, \quad z_{i k} \in D .
$$

Hence, (32) is proved for $i \neq k$. Consider now

Set

$$
\tilde{y}_{k k}=(k-1) ! y_{k}\left[z_{1}, \ldots, z_{k}\right] \text {. }
$$

$$
y_{k}(z)=z^{k-1} /(k-1) !+v_{k}(z) .
$$

By the linearity of the divided differences,

Hence,

$$
\begin{aligned}
\tilde{y}_{k k} & =(k-1) ! \frac{z^{k-1}}{(k-1) !}\left[z_{1}, \ldots, z_{k}\right]+(k-1) ! v_{k}\left[z_{1}, \ldots, z_{k}\right] \\
& =1+(k-1) ! v_{k}\left[z_{1}, \ldots, z_{k}\right] .
\end{aligned}
$$

$$
\tilde{y}_{k k}-1=(k-1) ! v_{k}\left[z_{1}, \ldots, z_{k}\right]=\lambda v_{k}^{(k-1)}\left(z_{k k}\right), \quad|\lambda|<1, \quad z_{k k} \in D .
$$

From (34) follows

$$
y_{k}^{(k-1)}\left(z_{k k}\right)-1=v_{k}^{(k-1)}\left(z_{k k}\right) .
$$

(35) and (36) imply (32) for $i=k$, and thus the proof of (32) is completed.

(31), (32) and Lemma 1 imply

$$
\lambda\left((\tilde{Y}-I)^{+}\right)<1 .
$$

Hence, by Lemma $2,|\tilde{Y}| \neq 0$ and we proved our theorem for the case $l=1, k_{1}=n$ of (29).

The proof for the other cases of (29) is established similarly. In the general case $\Omega$ is replaced by

$$
\Omega^{\prime}=\left(\begin{array}{lll}
y_{1}\left(z_{1}\right) & \cdots & y_{n}\left(z_{1}\right) \\
\vdots & & \vdots \\
y_{1}\left(z_{k_{1}}\right) & \cdots & y_{n}\left(z_{k_{1}}\right) \\
y_{1}^{\left(k_{1}\right)}\left(z_{k_{1}+1}\right) & \cdots & y_{n}^{\left(k_{1}\right)}\left(z_{k_{1}+1}\right) \\
\vdots & & \vdots \\
y^{\left(k_{1}\right)}\left(z_{k_{1}+k_{2}}\right) & \cdots & y_{n}^{\left(k_{1}\right)}\left(z_{k_{1}+k_{2}}\right) \\
\vdots & & \vdots \\
y_{1}^{\left(k_{1}+\cdots+k_{l-1}\right)}\left(z_{n}\right) & \cdots & y_{n}^{\left(k_{1}+\cdots+k_{l-1}\right)}\left(z_{n}\right)
\end{array}\right)
$$


and $\Omega_{1}^{\prime}$ is now obtained by taking divided differences separately in each set of rows with equal derivatives.

We end this section with an example. Consider the equation

$$
y^{\prime \prime}(z)-(m+1) y^{\prime}(z)+m y(z)=0, \quad m>1 .
$$

As can be easily verified, (37) is disconjugate exactly for

and is disfocal exactly for

$$
|z|<\pi /(m-1)=r_{m}
$$

$$
|z|<\log m / 2(m-1)=R_{m}
$$

(37) is thus strong disconjugate exactly for

$$
|z|<\min \left(r_{m}, R_{m}\right) \text {. }
$$

As for $m>\exp (2 \pi), r_{m}<R_{m}$ and for $m \leqq \exp (2 \pi), R_{m} \leqq r_{m}$, it follows that in general disfocality neither implies disconjugacy nor is implied by it.

As we stated before, $z_{0}$-absolute disconjugacy of (2) implies its (ordinary) disconjugacy. Our example shows that the converse is in general not true. Indeed, consider the system (2) corresponding to the equation (37). This system is disconjugate in the disk $|z|<R_{m}$ and, for $m>\exp (2 \pi)$, the equation (37) is not strong disconjugate there. Using Theorem 4 , it follows that for $m>\exp (2 \pi)$ no $z_{0}$, $\left|z_{0}\right|<R_{m}$, exists such that the system is $z_{0}$-absolute disconjugate in the disk $|z|<R_{m}$.

4. Sufficient conditions for strong disconjugacy of equations. In this section we apply Theorem 4 to Theorems $1-3$ and obtain sufficient conditions for strong disconjugacy of equations.

THEOREM 1'. Let $D$ be a bounded convex domain, and assume that the analytic functions $a_{l}(z), l=0, \ldots, n-1$, are regular and bounded in $D$. Let $z_{0} \in D$, and let $r=\sup \left|z-z_{0}\right|, z \in D$. For each $x, 0 \leqq x<r$, denote by $C(x)$ the intersection of the circle $\left|z-z_{0}\right|=x$ and $D$. Set

$$
m_{l}(x)=\sup _{z \in C(x)}\left|a_{l}(z)\right|, \quad l=0, \ldots, n-1, \quad 0 \leqq x<r .
$$

Let the nonnegative functions $p_{l}(x), l=0, \ldots, n-1$, be continuous in $0 \leqq x \leqq r$, and satisfy

$$
p_{l}(x) \geqq m_{l}(x), \quad l=0, \ldots, n-1, \quad 0 \leqq x<r .
$$

Let $u_{k}(x), k=1, \ldots, n, 0 \leqq x \leqq r$, be the solution of the real differential equation

$$
u^{(n)}(x)-p_{n-1}(x) u^{(n-1)}(x)-\cdots-p_{0}(x) u(x)=0, \quad 0 \leqq x \leqq r,
$$

satisfying

$$
u_{k}^{(i-1)}(0)=\delta_{i k}, \quad i=1, \ldots, n .
$$

Denote the corresponding Wronskian matrix by $\hat{U}(x)=\left(u_{k}^{(i-1)}(x)\right)_{1}^{n}$. If

$$
\lambda(\hat{U}(r))<2,
$$


then the complex differential equation

$$
y^{(n)}(z)+a_{n-1}(z) y^{(n-1)}(z)+\cdots+a_{0}(z) y(z)=0
$$

is strong disconjugate in $D$.

Proof. The differential equation (4) is equivalent to the differential systems (1) and (2) with $A(z)$ given by (3). Similarly the real majorant equation ( $\left.7^{\prime}\right)$ is equivalent to the real system (7) with

$$
P(x)=\left(\begin{array}{cccccc}
0 & 1 & 0 & & \cdots & 0 \\
0 & 0 & 1 & 0 & \ldots & 0 \\
\vdots & \vdots & & & & \vdots \\
0 & 0 & & \ldots & 0 & 1 \\
p_{0}(x) & p_{1}(x) & & \ldots & & p_{n-1}(x)
\end{array}\right) .
$$

$\hat{U}(x)$ is thus the fundamental solution of this system satisfying $\hat{U}(0)=I$. (9 $\left(9^{\prime}\right)$ implies, by Theorem 1 , that the system (2) is $z_{0}$-disconjugate in $D$. As we assumed that $D$ is convex, Theorem 4 gives thus the desired result.

We did not prove that the constant 2 on the right-hand side of $\left(9^{\prime}\right)$ is the best possible constant. However, 2 cannot be replaced by any number larger than $\exp (\pi / 4)=2.134 \cdots$. This follows by considering the equation

$$
y^{(n)}(z)+y^{(n-2)}(z)=0, \quad n \geqq 2 .
$$

$y(z)=\sin (z-\pi / 4)$ is a solution of (38) satisfying

$$
y\left(\frac{\pi}{4}\right)=y^{\prime}\left(-\frac{\pi}{4}\right)=y^{\prime \prime}\left(\frac{\pi}{4}\right)=\cdots=y^{(n-1)}\left((-1)^{n-1} \frac{\pi}{4}\right)=0 .
$$

(38) is thus not disfocal, and hence not strong disconjugate in any disk $D_{\varepsilon}=$ $\{z|| z \mid<(\pi / 4)+\varepsilon\}, \varepsilon>0$. Consider now (38) in $D_{\varepsilon}$, choose $z_{0}=0$ and set $p_{l}(x)$ $=m_{l}(x) .\left(7^{\prime}\right)$ becomes

$$
u^{(n)}(x)-u^{(n-2)}(x)=0, \quad 0 \leqq x \leqq \pi / 4+\varepsilon,
$$

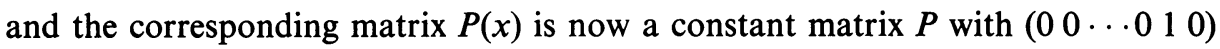
as its last row. $\lambda(P)=1$ and, as $\hat{U}(x)=\exp (x P)$, we obtain $\lambda(\hat{U}(\pi / 4))=\exp (\pi / 4)$.

THEOREM $2^{\prime}$. Let $D$ be a bounded convex domain, and let $r$ be the circumradius of $D$. Assume that the analytic functions $a_{l}(z), l=0, \ldots, n-1$, are regular and bounded in $D$ and denote

$$
\alpha_{l}=\sup _{z \in D}\left|a_{l}(z)\right|, \quad l=0, \ldots, n-1 .
$$

Set

$$
\rho=r / \log 2 \text {. }
$$

If

$$
\alpha_{n-1} \rho+\alpha_{n-2} \rho^{2}+\cdots+\alpha_{0} \rho^{n}<1,
$$

then the differential equation (4) is strongly disconjugate in $D$. 
Proof. Let $A(z)$ be defined by (3). The matrix $A=\left(\alpha_{i k}\right)_{1}^{n}$ of Theorem 2 becomes

$$
A=\left(\begin{array}{cccccc}
0 & 1 & 0 & & \cdots & 0 \\
0 & 0 & 1 & 0 & \ldots & 0 \\
\vdots & \vdots & & & & \vdots \\
0 & 0 & & \ldots & 0 & 1 \\
\alpha_{0} & \alpha_{1} & & \ldots & & \alpha_{n-1}
\end{array}\right) .
$$

The corresponding characteristic equation $|\lambda I-A|=0$ is given by

$$
\lambda^{n}-\alpha_{n-1} \lambda^{n-1}-\cdots-\alpha_{0}=0 .
$$

As we saw in $\S 3, y^{(n)}(z)=0$ is strongly disconjugate in the whole plane (and indeed (40) holds trivially for any $\rho$ ), so we may assume that $\sum_{l=0}^{n-1} \alpha_{l}>0$. Hence, the maximal characteristic value $\lambda(A)$ is positive and satisfies the equation (cf. [7, p. 259])

$$
\frac{\alpha_{n-1}}{\lambda(A)}+\frac{\alpha_{n-2}}{\lambda^{2}(A)}+\cdots+\frac{\alpha_{0}}{\lambda^{n}(A)}=1
$$

Clearly,

$$
f(x)=\alpha_{n-1} x+\alpha_{n-2} x^{2}+\cdots+\alpha_{0} x^{n}
$$

is, for $x>0$, a strictly increasing function of $x$. (39), (40) and (42) thus give

$$
r / \log 2<1 / \lambda(A) .
$$

Hence,

$$
r \lambda(A)<\log 2 .
$$

Theorem 2 thus implies that the system (2) is $z_{0}$-disconjugate in $D$, where $z_{0}$ denotes the circumcenter of $D$. Theorem 4 gives again the desired result.

The example given by (38) shows that the constant $\log 2=0.693 \cdots$, appearing in the definition (39) of $\rho$, cannot be replaced by any constant larger than $\pi / 4=0.785 \cdots$.

THEOREM $3^{\prime}$. Let $z_{0}$ be a point of the bounded convex domain $D$, and let $r=$ $\sup \left|z-z_{0}\right|, z \in D$. Let the analytic functions $a_{l}(z), l=0, \ldots, n-1$, be regular in $D$. For any $z \in D$, let $\int_{z_{0}}^{z}\left|a_{l}(\zeta) d \zeta\right|$ be taken along the segment from $z_{0}$ to $z$, and assume that these integrals are bounded uniformly in $z(z \in D)$. Denote

$$
c_{l}=\sup _{z \in D} \int_{z_{0}}^{z}\left|a_{l}(\zeta) d \zeta\right|, \quad l=0, \ldots, n-1 .
$$

If

$$
2 c_{n-1}+4 c_{n-2} r+\cdots+2^{n} c_{0} r^{n-1}<1,
$$

then the differential equation (4) is strongly disconjugate in $D$. 
Proof. Let $A(z)$ be defined by (3). The matrix $C=\left(c_{i k}\right)_{1}^{n}$ of Theorem 3 becomes

$$
C=\left(\begin{array}{cccccc}
0 & r & 0 & & \cdots & 0 \\
0 & 0 & r & 0 & \ldots & 0 \\
\vdots & \vdots & & & \vdots \\
0 & 0 & & \ldots & 0 & r \\
c_{0} & c_{1} & & \ldots & & c_{n-1}
\end{array}\right)
$$

The corresponding characteristic equation is

$$
\lambda^{n}-\lambda^{n-1} c_{n-1}-\lambda^{n-2} c_{n-2} r-\cdots-c_{0} r^{n-1}=0 .
$$

Assuming again that $\sum_{l=0}^{n-1} c_{l}>0$, it follows that $\lambda(C)>0$ satisfies

$$
\frac{c_{n-1}}{\lambda(C)}+\frac{c_{n-2} r}{\lambda^{2}(C)}+\cdots+\frac{c_{0} r^{n-1}}{\lambda^{n}(C)}=1
$$

(43) and (44) imply $2<1 / \lambda(C)$; i.e., we obtained the assumption (23) of Theorem 3. Theorem 3 and Theorem 4 give our assertion.

The example given by (38) shows that the powers of 2, appearing in (43), cannot be replaced by powers of any constant smaller than $4 / \pi=1.273 \cdots$.

5. A nonoscillation theorem. We conclude this paper with a nonoscillation theorem for the equation (4) in the unit disk. To facilitate the proof of this Theorem 5, we start with an elementary lemma; this Lemma 4 and the Peano-Baker expansion (11) give an assertion about the $z_{0}$-absolute disconjugacy of the system (2) in a subdomain $\Delta\left(z_{0}, \alpha\right)$ of the unit disk (Lemma 5); Lemma 5 and Theorem 4 yield then the desired result.

LEMMA 4. Let the analytic function $a(z)$ be regular for $|z|<1$, and denote

$$
m(x)=\max _{|z| \leqq x}|a(z)|, \quad 0 \leqq x<1 .
$$

For any angle $\alpha, 0<\alpha<\pi / 2$, and any point $z_{0}, 0<\left|z_{0}\right|<1$, let the domain $\Delta\left(z_{0}, \alpha\right)$ be given by

$$
\Delta\left(z_{0}, \alpha\right)=\left\{z|| z \mid<1 \text { and }\left|\arg \left(z-z_{0}\right)-\arg \left(z_{0}\right)\right|<\alpha\right\} .
$$

For any two points $z_{0}, z$, satisfying $0<\left|z_{0}\right|<1$ and $z \in \Delta\left(z_{0}, \alpha\right)$, the inequality

$$
\int_{z_{0}}^{z}|a(\zeta) d \zeta| \leqq \frac{1}{\cos \alpha} \int_{\left|z_{0}\right|}^{|z|} m(x) d x
$$

holds. Here the first integral is taken along the segment $\left[z_{0}, z\right]$ from $z_{0}$ to $z$. 
Proof. Let $\zeta$ and $\zeta+\Delta \zeta$ be on the segment $\left[z_{0}, z\right]$ such that $|\zeta|<|\zeta+\Delta \zeta|$ $(=|\zeta|+\Delta|\zeta|)$. Let $\beta=\left|\arg \left(z-z_{0}\right)-\arg \left(z_{0}\right)\right|$ and $\gamma=\left|\arg \left(z-z_{0}\right)-\arg (\zeta)\right|$. Then $0 \leqq \gamma \leqq \beta<\alpha$. Clearly,

$$
|\Delta \zeta| \leqq \frac{1}{\cos \gamma} \Delta|\zeta|<\frac{1}{\cos \alpha} \Delta|\zeta|
$$

Hence,

$$
\int_{z_{0}}^{z}|a(\zeta) d \zeta| \leqq \int_{z_{0}}^{z} m(|\zeta|)|d \zeta| \leqq \frac{1}{\cos \alpha} \int_{\left|z_{0}\right|}^{|z|} m(x) d x
$$

Lemma 5. Let the analytic functions $a_{i k}(z), i, k=1, \ldots, n,\left(A(z)=\left(a_{i k}(z)\right)_{1}^{n}\right)$ be regular for $|z|<1$, and set

$$
m_{i k}(x)=\max _{|z| \leqq x}\left|a_{i k}(z)\right|, \quad i, k=1, \ldots, n, \quad 0 \leqq x<1 .
$$

Assume that

$$
\int_{0}^{1} m_{i k}(x) d x<\infty, \quad i, k=1, \ldots, n .
$$

Let $\alpha, 0<\alpha<\pi / 2$, be given. Then there exists a number $x_{1}, 0<x_{1}<1$, dependent only on $\alpha$ and on the functions $m_{i k}(x)$, such that the differential system (2) is, for every $z_{0}$ in the ring

$$
x_{1} \leqq\left|z_{0}\right|<1,
$$

$z_{0}$-absolute disconjugate in $\Delta\left(z_{0}, \alpha\right)$.

Proof. Given $\alpha, 0<\alpha<\pi / 2$, and the functions $m_{i k}(x)$ satisfying (49), we choose $x_{1}$ such that

$$
\int_{x_{1}}^{1} m_{i k}(x) d x \leqq \frac{1}{2 n} \cos \alpha, \quad i, k=1, \ldots, n .
$$

Let $z_{0}$ satisfy (50), and let $W(z)=\left(w_{i k}(z)\right)_{1}^{n}$ be the solution of (2) satisfying

$$
W\left(z_{0}\right)=I \text {. }
$$

$\left(11^{\prime}\right)$ gives

(52) $\left|w_{i k}(z)-\delta_{i k}\right| \leqq \int_{z_{0}}^{z}\left|a_{i k}(\zeta) d \zeta\right|+\sum_{j=1}^{n} \int_{z_{0}}^{z}\left|a_{i j}(\zeta)\right| \int_{z_{0}}^{\zeta}\left|a_{j k}\left(\zeta_{1}\right) d \zeta_{1}\right||d \zeta|+\cdots$

Let now $z$, and hence also $\zeta, \zeta_{1}, \ldots$, be in $\Delta\left(z_{0}, \alpha\right)$. Using Lemma 4 , we obtain by (50) and (51) that

$$
\int_{z_{0}}^{z}\left|a_{i k}(\zeta) d \zeta\right|<\frac{1}{2 n}, \quad \int_{z_{0}}^{\zeta}\left|a_{j k}\left(\zeta_{1}\right) d \zeta_{1}\right|<\frac{1}{2 n}, \ldots
$$

(52) and (53) give, for $i, k=1, \ldots, n$ and $z \in \Delta\left(z_{0}, \alpha\right)$,

$$
\left|w_{i k}(z)-\delta_{i k}\right|<\frac{1}{2 n}+n\left(\frac{1}{2 n}\right)^{2}+n^{2}\left(\frac{1}{2 n}\right)^{3}+\cdots=\frac{1}{n} .
$$


It follows that for every choice of $n^{2}$ points $z_{i k}$ in $\Delta\left(z_{0}, \alpha\right)$

$$
\left|w_{i k}\left(z_{i k}\right)-\delta_{i k}\right|<\frac{1}{n}, \quad i, k=1, \ldots, n .
$$

Hence,

$$
\lambda\left(\left(\left|w_{i k}\left(z_{i k}\right)-\delta_{i k}\right|\right)_{1}^{n}\right)<1
$$

and we thus proved Lemma 5.

THEOREM 5. Let the analytic functions $a_{l}(z), l=0, \ldots, n-1$ be regular for $|z|<1$, and set

$$
m_{l}(x)=\max _{|z| \leqq x}\left|a_{l}(z)\right|, \quad 0 \leqq x<1, \quad l=0, \ldots, n-1
$$

If

$$
\int_{0}^{1} m_{l}(x) d x<\infty, \quad l=0, \ldots, n-1,
$$

then no nontrivial solution of the differential equation (4) can have an infinite number of zeros in $|z|<1$.

Proof. Assume, by contradiction, that a solution $y(z) \not \equiv 0$ of (4) has an infinite number of zeros in $|z|<1$, and let $\exp (i \theta)$ be an accumulation point of these zeros. For any given $\alpha, 0<\alpha<\pi / 2$, and $x_{1}, 0<x_{1}<1, y(z)$ will have infinite many zeros in $\Delta\left(z_{0}, \alpha\right)$ with $z_{0}=x_{1} \exp (i \theta)$. But the system (2), with $A(z)$ given by (3), is, by Lemma $5, z_{0}$-absolute disconjugate in $\Delta\left(z_{0}, \alpha\right)$. By Theorem 4 , the equation (4) is thus strongly disconjugate in $\Delta\left(z_{0}, \alpha\right)$, and $y(z)$ has therefore at most $n-1$ zeros in this domain.

It is not difficult to show that, under the assumptions of Theorem 5, there exists a number $N$, dependent only on the $n$ functions $m_{l}(x)$, such that no solution of (4) has more than $N$ zeros in $|z|<1$. This follows by choosing a fixed $\alpha$, say $\alpha=\pi / 4$, and covering the unit disk by a finite number $q$ of eccentric wedges of the form

$$
\tilde{\Delta}\left(z^{*}, \alpha\right)=\left\{z|| \arg \left(z-z^{*}\right)-\arg \left(z^{*}\right) \mid<\alpha \text { and }|z|-\left|z^{*}\right|<1-x_{1}\right\} .
$$

Any solution $y(z)$ has at most $n-1$ zeros in each wedge, hence $N=q(n-1)$.

Theorem 5 implies a theorem of Nehari [8, p. 331] stating that (4) is nonoscillatory in the unit disk if

$$
\lim _{r \rightarrow 1} \int_{0}^{2 \pi}\left|a_{l}\left(\mathrm{re}^{i \theta}\right)\right| d \theta<\infty, \quad l=0, \ldots, n-2
$$

and

$$
\lim _{r \rightarrow 1} \int_{0}^{2 \pi}\left|a_{n-1}\left(\mathrm{re}^{i \theta}\right)\right| d \theta<1 .
$$

Indeed, if $a_{l}(z)$ belongs to $H^{1}$ (i.e., satisfies (55)), then $\left(49^{\prime}\right)$ holds [4, p. 236]. 


\section{REFERENCES}

1. P. J. Davis, Interpolation and approximation, Blaisdell, New York, 1965.

2. H. G. Eggleston, Convexity, Cambridge Tracts in Math and Math Phys., No. 47, Cambridge Univ. Press, New York, 1958.

3. F. R. Gantmacher, The theory of matrices, Vol. II, Chelsea, New York, 1959.

4. G. H. Hardy, J. E. Littlewood and G. Pólya, Inequalities, 2nd ed., Cambridge Univ. Press, New York, 1952.

5. E. L. Ince, Ordinary differential equations, Dover, New York, 1926; reprint, 1944.

6. D. London and B. Schwarz, On the radius of disfocality of a class of polynomials, J. Math. Anal. Appl. 20 (1967), 521-533.

7. Z. Nehari, "On an inequality of Lyapunov" in Studies in mathematical analysis and related topics, essays in honour of George Pólya, pp. 256-261, Stanford Univ. Press, Stanford, Calif., 1962.

8. - On the zeros of solutions of nth order linear differential equations, J. London Math. Soc. 39 (1964), 327-332.

9. —_ Some function-theoretic aspects of linear second-order differential equations, J. Analyse Math. 18 (1967), 259-276.

10. N. E. Nörlund, Leçons sur les séries d'interpolation, Gauthier-Villars, Paris, 1926.

11. A. M. Ostrowski, Positive matrices and functional analysis, Proc. Advanced Seminar, Math. Res. Center, U.S. Army, Univ. of Wisconsin Press, Madison, 1964, pp. 81-101.

12. B. Schwarz, Disconjugacy of complex differential systems, Trans. Amer. Math. Soc. 125 (1966), 482-496.

TeChNion, IsRael Institute of TeChNology, HaIFA, IsRael 